\title{
Materiales vitrocerámicos a partir de lodos procedentes de una estación de depuración de aguas residuales urbanas (en la Ciudad de El-Sadat, Egipto).
}

\author{
M. GARCIA-VALLES', M.H. ALY ${ }^{2}$, E. EL- FADALY ${ }^{2}$, H.S. HAFEZ ${ }^{2}$, J. NOGUÉS ${ }^{1}$ Y S. MARTINEZ ${ }^{1}$ \\ 'Dpt. Cristal·lografia, Mineralogia i Dip. Minerals, Facultat de Geologia, Universitat de Barcelona (UB), 08028 Barcelona (España) \\ ²Environmental Studies and Research Institut, Minufiya University, Sadat City, Minufiya (Egypt) maitegarciavalles@ub.edu
}

\begin{abstract}
La depuración de las aguas residuales urbanas es fuente de importantes volúmenes de lodos los cuales es preciso gestionar. En este trabajo se expone la posibilidad de aplicarles un proceso de gestión basado en la vitrificación y mediante el cual, además de inertizar los elementos contaminantes presentes en los lodos, se obtiene un material vitrocerámico con un importante valor añadido. Partiendo de la caracterización química (FRX), mineralógica (DRX) y térmica (ATD-TG) de estos lodos, se determina la formulación del vidrio original y su poder energético. Mediante ATD-TG, dilatometría y MEB, se ha determinado la temperatura de máxima velocidad de nucleación y la temperatura de crecimiento así como la morfología y tamaño de los núcleos formados que, en este caso, son nanométricos. En definitiva, se ha comprobado como la gestión de los residuos sólidos urbanos mediante la vitrificación es una alternativa a los vertederos y a la incineración.
\end{abstract}

Palabras calve: vitrocerámica, lodo depuradora, valorización, vidrio

Production of glass-ceramic materials obtained from sewage sludge treatment plant for urban wastewater in El-Sadat city (Egypt).

Urban wastewater security is a source of significant volumes of sludge which is to be managed. This paper presents the possibility of applying a management process based on vitrification and by which, in addition to inert contaminants in sludge, you get a ceramic material with a significant added value. As a base of different analysis of the sludges, chemical $(\mathrm{XRF})$, mineralogical (XRD) and thermal (DTA-TG), we obtain the original glass composition and its energy. By DTATG, dilatometry and SEM, it was determined the temperature of maximum nucleation rate, growth temperature and the morphology and size of developed nuclei, in this case are nanoscale. Briefly, it was found as the management of municipal solid waste by vitrification as an alternative to landfills and incineration.

Key words: glass ceramic, sludge, glass, valorization

\section{INTRODUCCIÓN}

Una parte importante de los problemas medioambientales están relacionados con la gestión de residuos, concretamente con la utilización y / o almacenamiento de los lodos producto del tratamiento de residuos municipales e industriales, debido al volumen que se generan diariamente así como a los elementos tóxicos y peligrosos, particularmente metales pesados, que pueden contener en función de la diversidad de industrias que generan el lodo. Así mismo, cabe considerar que su almacenamiento, tratamiento y transporte conlleva además costes económicos, involucrado también un elevado consumo energético. Hace falta pues buscar soluciones medioambientalmente correctas que permitan valorizar el residuo obteniendo un producto útil y con un valor añadido, contribuyendo así con la sostenibilidad. El problema del almacenamiento y uso de los lodos de depuradora va más allá de las fronteras y/o países.

En la estrategia de gestión de los residuos del $6^{\circ}$ Programa de Actuación Medioambiental de la Unión
Europea (6EAP), se contemplan patrones de producción y consumo sostenibles (producción Eco-eficiente) encaminándose a reducir el impacto ambiental de las fuentes utilizadas y conseguir estrategias de desarrollo sostenible. Planteando una jerarquía de gestión para la gestión de los residuos, fundamentada en: a) prevención de residuos, b) reutilización de residuos y componentes, c) reciclaje de materiales, d) recuperación de energía y e) deposición en vertederos en las mejores condiciones medioambientales posibles.

La utilización masiva de materias primas y recursos naturales afecta directamente a la conservación del medio ambiente, su impacto puede evitarse o reducirse mediante la reutilización o el reciclado de estos productos en su fase de residuo, así como con el diseño eco-eficiente. Hay que tener en cuenta, que todo producto manufacturado genera un impacto ambiental el cual se inicia ya en el proceso de extracción de las materias primas, continuando en el proceso de fabricación, así como en su ciclo de vida 
Actualmente, en Egipto los lodos de depuradora también constituyen un problema, ya que el aumento de la población ha sido muy alto, se ha triplicado en los últimos 50 años. Este incremento se prevé que dentro de quince años sea de 96 millones. Mayoritariamente, la población egipcia vive alrededor del Nilo, se concentran principalmente en el valle y delta del Nilo, se trata de áreas rurales. Paralelamente al aumento de la población ha incrementado también el volumen industrial lo cual conlleva a la generación de un mayor volumen de lodos en las depuradoras. Están en funcionamiento 303 depuradoras con una capacidad de $11.8510^{6} \mathrm{~m}^{3}$ / día. En Egipto, en el año 2002 se generaron de 12 a $15 \mathrm{~kg} /$ año por habitante de materia sólida procedente de los lodos de depuradora, estimándose que hoy día es de $1.742 .758,2$ ton/ año de lodo seco, a la expectativa que en el año 2020 haya incrementado en 2 millones de toneladas (1). Estadísticamente, se produce aproximadamente $12-15 \mathrm{~kg} /$ año de lodo por habitante. Corresponde a una producción diaria de 35 - $40 \mathrm{~g}$. En la actualidad, el destino principal de estos lodos es su vertido en el desierto. Este panorama deja la puerta abierta a unas perspectivas de crecimiento desmesurado de los lodos en un futuro. Por ello, en la línea de las orientaciones expresadas en el 6EAP es necesario ir avanzando en la investigación de propuestas para reducir y/o eliminar los lodos generados revalorizándolos.

En los últimos años, uno de los principales temas de investigación ha sido la forma de obtener el vidrio y materiales cerámicos de los lodos y residuos industriales (2-9), así como de otras aplicaciones dentro de estos campos (10). Estos materiales vitroceramicos presentan una elevada resistencia a la compresión, flexión y abrasión (9) pudiendo estimar que la aplicabilidad de este tipo de materiales va en la línea de ser utilizados como "roca sintética" pudiendo ser empleada como pavimento industrial y urbano, material de cubierta (tejados de pizarra), revestimientos, etc. Algunos autores utilizan la vitrificación para la estabilización y la reutilización de las cenizas volantes generadas en los procesos de incineración (11-17). También se ha propuesto la vitrificación, como una tecnología de estabilización de residuos, radiactivos $(18,19)$. En el proceso de vitrificación el residuo pasa a ser un vidrio y / o vitrocerámico estable (8) apto para la obtención de productos para fines comerciales, de manera que se revaloriza un residuo y se resuelve un problema medioambiental. La vitrificación admite el uso de residuos de distintos tipos teniendo en cuenta que siempre hay que controlar el quinismo de los componentes que intervienen. El contenido orgánico de los residuos también se puede usar como fuente de energía auxiliar, esta energía de reciclaje representa un ahorro de $\mathrm{CO}_{2}$ de Kyoto. Las fases inorgánicas tóxicas se incorporan en el vidrio estabilizándose por completo en la estructura del nuevo material vítreo o vitrocerámico.

El objetivo de este trabajo es el estudio y caracterización de los lodos de la estación depuradora de aguas residuales (EDAR) procedentes de la depuradora de El-Sadat (Minufiya, Egipto) así como la formulación, obtención y caracterización de un vidrio obtenido a partir de dicho lodo para su posible aplicación como material vitrocerámico en la industria de la construcción. Obteniéndose así un producto con valor añadido. En función de la composición del lodo se evalúa la necesidad de emplear otro tipo de residuo para poder formular adecuadamente un vidrio.

\section{MATERIAL Y MÉTODOS}

Los lodos EDAR empleados en este estudio corresponden a la depuradora de Sadat City (Egipto). Se trata de una depuradora que procesa aguas municipales e industriales, ya que se encuentra emplazada en una zona que, desde el punto de vista de Egipto, se considera relativamente industrial. Se trata de un área desértica en la que hay abundantes industrias de acero, hierro, pinturas, cerámicas, químicas, fertilizantes, etc. La depuradora se localiza al este de la provincia de Al-Minufiya, la cual tiene una extensión de $500 \mathrm{~km}^{2}$ y una población de aproximadamente 600.200 habitantes, se encuentra a unos $93 \mathrm{~km}$ de El Cairo. Aproximadamente más de unos $30.000 \mathrm{~m}^{3} /$ día de aguas residuales se gestionan en el tanque de oxidación. Se estima que en el tanque de oxidación se producen diariamente 6,03 ton/día de lodo seco.

Los lodos utilizados se recogieron del tanque de oxidación, previamente se había eliminado el papel, el plástico, la madera y la arena, en la primera etapa de tratamiento físico.

La composición química del lodo se ha determinado mediante fluorescencia de rayos- $x$ (FRX), utilizando un espectrómetro secuencial de rayos-X Phillips PW 2400. Para los elementos mayoritarios se han realizado perlas de tetraborato de litio con una disolución 1/20. Mientras que para los minoritarios se han preparado pastillas.

La mineralogía del lodo, del vidrio original y del vidrio tratado térmicamente se ha determinado mediante difracción de rayos-X (DRX) utilizando un difractómetro de geometría Bragg-Brentano PANalytical X'Pert system, con monocromador de grafito, rendija automática, radiación $\mathrm{K} \alpha$ del $\mathrm{Cu}(\lambda=1.54061 \AA ̊)$, potencia de trabajo $45 \mathrm{kV}-40 \mathrm{~mA}$, rango de barrido $4-100^{\circ} 2 \theta$ con paso de $0.017^{\circ} 2 \theta$ y tiempo de medida de $50 \mathrm{~s}$. El software específico utilizado en la identificación y en la valoración semicuantitativa de las fases ha sido XPert Graphics Identify Philips.

El estudio térmico del lodo y del vidrio se ha realizado mediante análisis térmico diferencial sincrónicamente con el termogravimétrico (ATD-TG), utilizando un equipo Netzsch (STA 409C), con atmósfera de aire con un flujo de $80 \mathrm{ml} /$ min, con una velocidad de calentamiento de $10^{\circ} \mathrm{C} / \mathrm{min}$ desde temperatura ambiente hasta $1.200^{\circ} \mathrm{C}$. Se han utilizado $70 \mathrm{mg}$ de muestra, con una granulometría $<100 \mu \mathrm{m}$, con un crisol de $\mathrm{Al}_{2} \mathrm{O}_{3}$ y como patrón alúmina de referencia Perkin Elmer 0419-0197. Este análisis ha permitido ver el comportamiento térmico del lodo y medir su poder calorífico (PCI); y en el caso del vidrio establecer el inicio de la nucleación y la temperatura de crecimiento.

La temperatura de transición del vidrio $(\mathrm{Tg})$ se ha determinado con un dilatómetro Linsei L76/1550, calentando a $10^{\circ} \mathrm{C} / \mathrm{min}$ en atmósfera de aire, utilizando probetas de $5 \times 5 \times 30 \mathrm{~mm}$.

La caracterización morfológica y composicional de vidrio original tratado térmicamente se ha estudiado mediante microscopia electrónica de barrido (MEB). Se han utilizado dos microscopios distintos en función de la resolución a la que era necesario llegar: Hitachi H-4100FE filamento de emisión de campo, cátodo frío, con una resolución de 1,5 nm; y Stereoscan S-360 con detector de dispersión de energías de rayos-X (EDS) (INCA Energy 200 Oxford Instruments). En ambos casos las muestras fueron recubiertas con carbón en un equipo de vaporización por "sputtering". 


\section{RESULTADOS Y DISCUSIÓN}

La composición química del lodo EDAR de El-Sadat se recoge en la tabla I. Como se aprecia, el elemento mayoritario del lodo es la sílice; en menor cantidad le siguen el Al, el Fe y el Ca, y en menor proporción se observa el $\mathrm{Na}$, el $\mathrm{P}$, el $\mathrm{K}$ y el $\mathrm{Mg}$ por orden de contenido. Finalmente, en una proporción inferior a uno están el Ti y el Mn. En el diagrama de Le Maitre (20) de la figura 1, correspondiente al contenido en sílice vs. el total de álcalis, vemos que el lodo de El-Sadat queda situado dentro del campo perteneciente a la composición de un basalto. Aunque a diferencia de él, la proporción de fósforo es mayor. Por lo que se refiere a los elementos minoritarios, en general se encuentran en muy poca proporción (Tabla I), siendo superior al límite permitido para ser utilizado el lodo en agricultura (21 y 22) en el caso del $\mathrm{Cr}$, el $\mathrm{Zn}$ y el $\mathrm{Pb}$, los cuales presentan valores superiores a los permitidos. Así pues, con la legislación Europea estos lodos no se podrían utilizar directamente en un suelo agrícola.

El control cualitativo de las materias primas en la obtención de un vidrio, sea natural o bien como en este caso a partir de un residuo, es básica, ya que la eficiencia del proceso de fusión, su comportamiento, sus propiedades y la misma calidad del vidrio depende de dicha composición. Por lo tanto, en los resultados presentados en la tabla 1 se especifica el análisis correspondiente al muestreo, que es representativo del momento concreto en el que se realizó la toma, en el caso de tenerse que aplicar industrialmente seria necesario un estudio estadístico y temporal a lo largo de un año para poder evaluar la variabilidad de las composiciones de dichos lodos.

Desde un punto de vista mineralógico, el lodo EDAR esta constituido por un $33 \%$ de cuarzo $\left(\mathrm{SiO}_{2}\right)$, un $29 \%$ de albita $\left(\mathrm{Na}\left[\mathrm{Si}_{3} \mathrm{AlO}_{8}\right]\right)$, un $10 \%$ de ortosa $\left(\mathrm{K}\left[\mathrm{Si}_{3} \mathrm{AlO}_{8}\right]\right)$, un $13 \%$ de yeso $\left(\mathrm{Ca}\left[\mathrm{SO}_{4}\right] \cdot \mathrm{H}_{2} \mathrm{O}\right)$ y un $15 \%$ de caolinita $\left(\mathrm{Al}_{4}\left[\mathrm{Si}_{4} \mathrm{O}_{10}\right](\mathrm{OH})_{8}\right)$. El cuarzo y los feldespatos son pues las fases minerales mayoritarias. En la figura 2 se observa el espectro de DRX correspondiente al lodo, en el que se han señalado solo los picos de máxima reflexión de cada una de las fases, utilizando las siglas de abreviación internacionales de los minerales. La cuantificación de cada una de las fases hay que considerarla semicuantitativa ya que se ha realizado directamente con el software de interpretación.

Una vez el lodo ha sido caracterizado química y mineralógicamente se ha procedido a analizar su comportamiento térmico. En la figura 3 se observa el diagrama ATD-TG del lodo EDAR. En la curva correspondiente al ATD se aprecian dos picos exotérmicos, uno a $296,6^{\circ} \mathrm{C}$ y otro a $433,8^{\circ} \mathrm{C}$ correlacionables con las perdidas de peso registradas en la TG; y un pico endotérmico a aproximadamente $100^{\circ} \mathrm{C}$ directamente atribuible a la perdida de agua. En la curva TG se aprecian tres pérdidas de peso importantes de 1,93\%, $11,88 \%$ y $20,97 \%$, los valores de las cuales son mayores con el incremento de la temperatura. Las dos primeras pérdidas de peso están claramente relacionadas con la oxidación de la materia orgánica.

Desde un punto de vista energético, el ATD se ha utilizado para calcular el poder calorífico (PCI) que podríamos extraer del lodo EDAR en el proceso de su vitrificación. Para deducir el PCI se ha considerando un gran exotérmico que incluye los dos anteriormente determinados (Figura 3). El área de este amplio pico exotérmico, tal y como se observa en la gráfica, es de $2.506 \mu \mathrm{Vs} / \mathrm{mg}$ a partir de este parámetro y la
TABLA I. COMPOSICIÓN QUÍMICA DEL LODO DE LA DEPURADORA EN PORCENTAJE EN PESO, DETERMINADO MEDIANTE FXR.

Composición química $(\%$ peso)

\begin{tabular}{|c|c|c|c|}
\hline \multicolumn{2}{|c|}{ Elementos mayoritarios } & \multicolumn{2}{|c|}{ Elementos minoritarios } \\
\hline $\mathrm{SiO}_{2}$ & 48,70 & $\mathrm{Cr}_{2} \mathrm{O}_{3}$ & $0,105^{*}$ \\
\hline $\mathrm{Al}_{2} \mathrm{O}_{3}$ & 15,90 & $\mathrm{ZnO}$ & $1,202^{*}$ \\
\hline $\mathrm{Fe}_{2} \mathrm{O}_{3}$ & 9,66 & $\mathrm{ZrO}_{2}$ & 0,843 \\
\hline $\mathrm{CaO}$ & 4,71 & $\mathrm{BaO}$ & $<0,11$ \\
\hline $\mathrm{MgO}$ & 1,16 & $\mathrm{PbO}$ & $0,171^{*}$ \\
\hline $\mathrm{K}_{2} \mathrm{O}$ & 1,30 & $\mathrm{SO}_{3}$ & 5,199 \\
\hline $\mathrm{Na}_{2} \mathrm{O}$ & 1,39 & & \\
\hline $\mathrm{P}_{2} \mathrm{O}_{5}$ & 1,67 & & \\
\hline $\mathrm{TiO}_{2}$ & 0,91 & & \\
\hline$P C$ & 6,97 & & \\
\hline
\end{tabular}

* Valor superior al límite establecido para su uso en agricultura (CEE, 1986 y BOE, 1990)

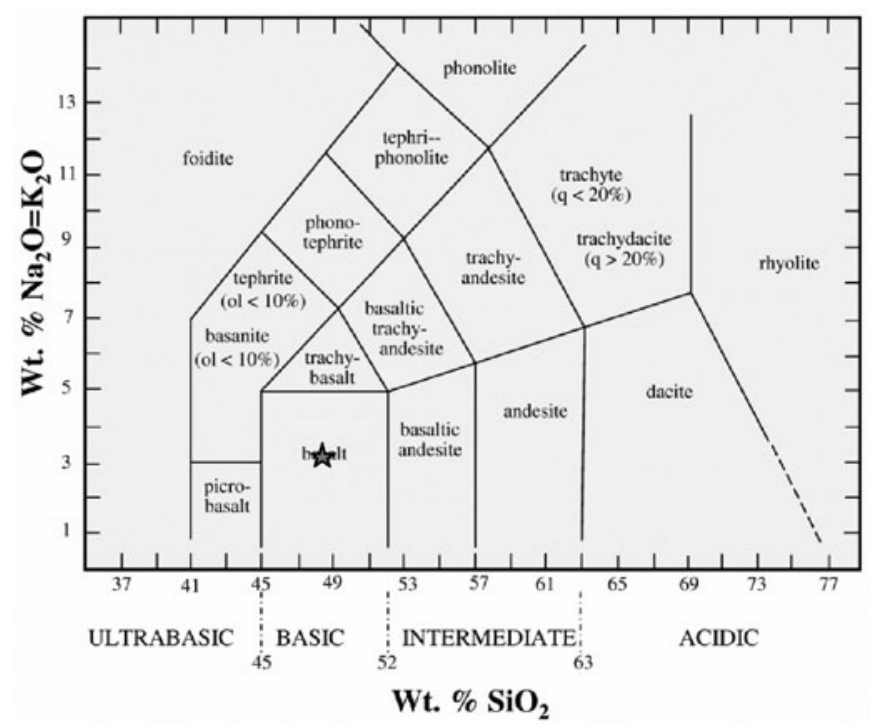

Figura 1. Diagrama de Le Maitre et al. (2002) del contenido en sílice vs. el total de álcalis situando en donde se ha situado el lodo de El-Sadat.

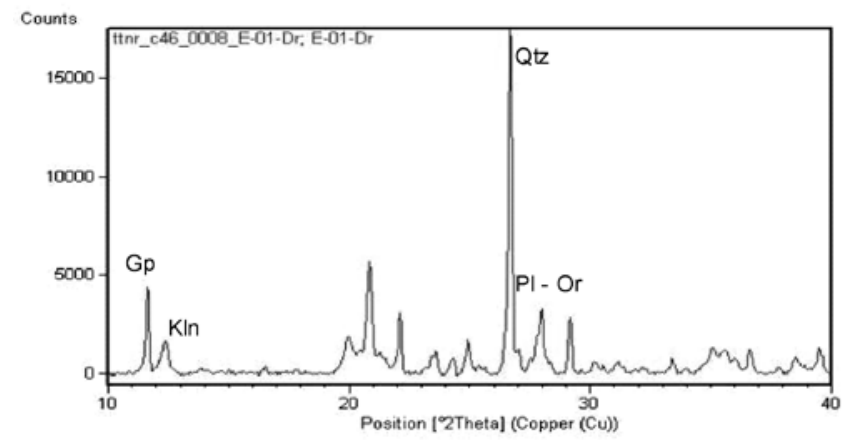

Figura 2. Difractograma del lodo de El-Sadat en donde están marcados los picos principales de cada una de las fases: Qtz cuarzo, Pl plagioclasas, Or ortosa, Gp yeso y Kln caolinita. 


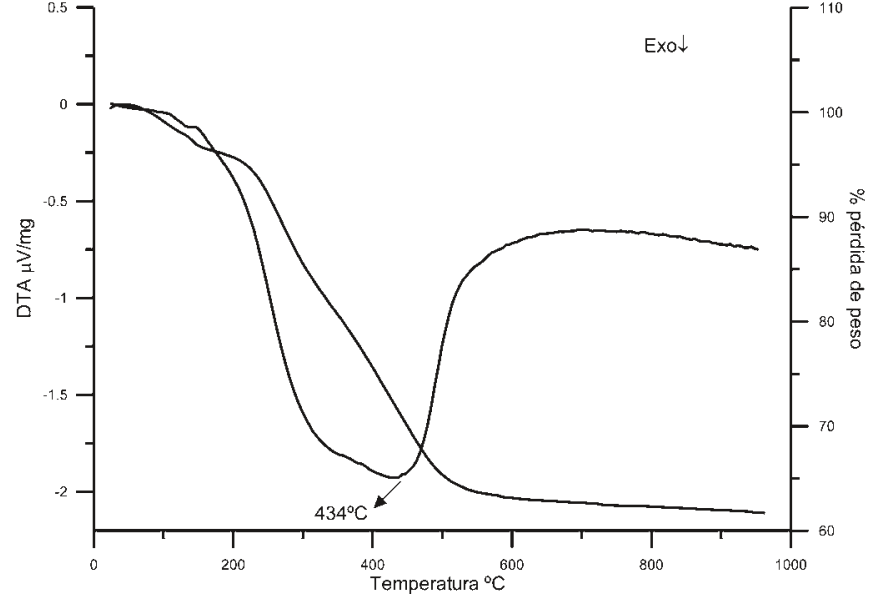

Figura 3. ATD-TG correspondiente al lodo EDAR de El-Sadat.

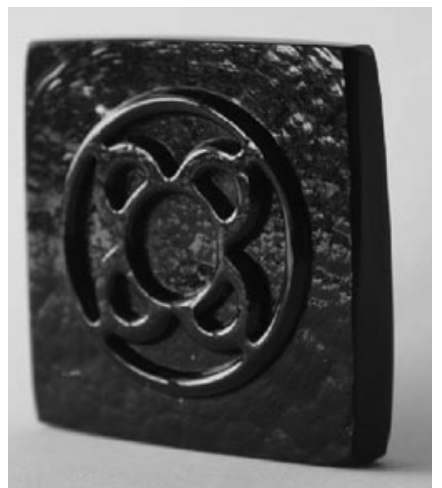

Figura 4. Vidrio original producto de la vitrificación del lodo de El-Sadat.

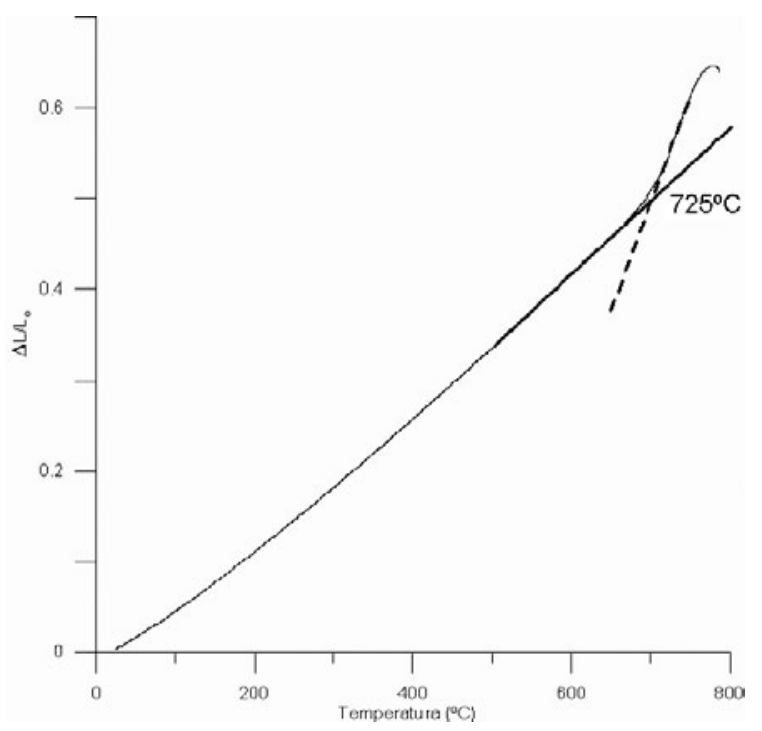

Figura 5. Curva dilatomètrica correspondiente al vidrio original procedente del lodo EDAR. comparación con una recta patrón, obtenida a partir de los PCI de varias depuradoras, determinados de forma convencional, comparados con los picos exotérmicos obtenidos por ATD, de estos mismos lodos, se ha calculado el PCI, siendo de 13,01 $\mathrm{MJ} / \mathrm{Kg}$. Este valor nos da idea de la efectividad energética del proceso, la cual entraría dentro de las consideraciones establecidas y recomendadas en el 6EAP.

A partir de la composición química del lodo se ha formulado la mezcla a utilizar para obtener el vidrio original. El lodo pasa a ser la materia prima básica en la obtención del vidrio. Se ha mezclado un $93 \%$ de lodo previamente calcinado a $950{ }^{\circ} \mathrm{C} / 2 \mathrm{~h}$, con un $2 \%$ de $\mathrm{Na}_{2}\left(\mathrm{CO}_{3}\right)$ y un $5 \%$ de $\mathrm{Ca}\left(\mathrm{CO}_{3}\right)$ como agentes aditivos necesarios para llegar a una temperatura de fusión óptima para colar el vidrio adecuadamente. La fusión se ha realizado en un horno de super-kantal a una temperatura de $1450^{\circ} \mathrm{C}$ en un crisol de alúmina durante 8 horas, después se ha colado sobre un molde metálico con un dibujo modernista, previamente calentado y se ha dejado enfriar en un horno que estaba a $700{ }^{\circ} \mathrm{C}$ para el recocido del vidrio original (figura 4). A esta temperatura de fusión, se destruyen los compuestos orgánicos de manera que deja de ser un "material" potencialmente nocivo. Por otra parte, los metales con baja presión de vapor, como el $\mathrm{Hg}$ y el $\mathrm{Pb}$, se evaporan y quedan atrapados en los equipos de control de los gases de salida, en este caso solo nos preocuparía el $\mathrm{Pb}$ ya que no se había determinado la presencia de $\mathrm{Hg}$ en la muestra objeto de estudio (Tabla I). Por lo que se refiere a los demás metales pesados y nocivos determinados en el lodo se estabilizan de manera permanente en la matriz del vidrio (9).

El ATD del vidrio original ha permitido determinar su temperatura de crecimiento cristalino en $938^{\circ} \mathrm{C}$. Su temperatura de transición $\left(\mathrm{T}_{\mathrm{g}}\right)$ ha sido determinada también mediante la curva dilatométrica, obteniéndose un valor de $725^{\circ} \mathrm{C}$ (Figura 5).

A partir de este punto, el tratamiento térmico del vidrio original (ATD) triturado y tamizado entre 400 y $500 \mu \mathrm{m}$, ha permitido establecer el intervalo de temperatura de nucleación, la temperatura de máxima nucleación y el crecimiento cristalino. Parámetros necesarios para la fabricación del vitrocerámico. Para ello se ha sometido la muestra de vidrio original al siguiente programa de calentamiento: en primer lugar se ha calentado a una velocidad de $10^{\circ} \mathrm{C} / \mathrm{min}$ hasta la temperatura de nucleación escogida dentro del intervalo de 600 a $825^{\circ} \mathrm{C}$ durante un periodo de 3 horas. Posteriormente, sin sacar la muestra del ATD, se prosigue el calentamiento a $15^{\circ} \mathrm{C} / \mathrm{min}$ hasta una temperatura superior a la temperatura de crecimiento cristalino (pico exotérmico del ATD).

La temperatura de nucleación se puede obtener mediante el análisis de la temperatura del pico exotérmico $\left(\mathrm{T}_{\mathrm{ex}}\right)$ que está relacionado con el número de núcleos por unidad de volumen que se formó en el cristal (N). Esta relación se establece por la expresión:

$$
\ln \mathrm{N}=\frac{\mathrm{m} * \mathrm{E}_{\mathrm{c}}}{\mathrm{R} * \mathrm{~T} \text { ex }}+\mathrm{Ct}
$$

$\mathrm{R}=$ cte. general de los gases

$\mathrm{E}_{\mathrm{c}}=$ Energía de activación.

$\mathrm{m}=$ Cte. dependiente de la dimensionalidad del crecimiento.

Por lo tanto $1 / \mathrm{T}_{\mathrm{ex}}$ es proporcional a $\mathrm{N}$. 


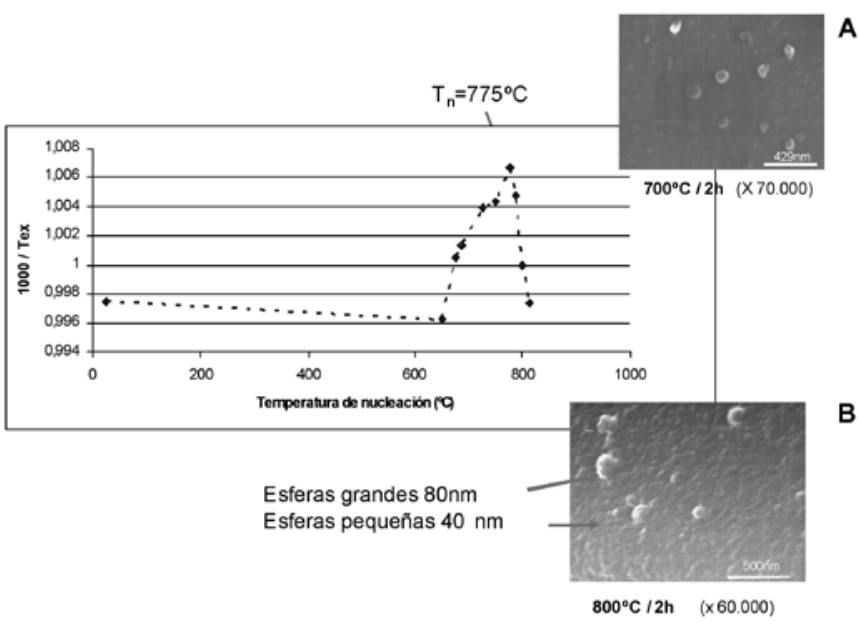

Figura 6. Grafica que nos relaciona la inversa de la temperatura de crecimiento cristalino (Tex) con la temperatura de nucleación (Tn) del vidrio original. En las figuras A y B se muestran las imágenes de SEM de los núcleos formados en el vidrio a 700 y $800^{\circ} \mathrm{C}$ respectivamente.

Si representamos $1000 / \mathrm{T}_{\text {ex }}$ en función de la temperatura de nucleación $\left(\mathrm{T}_{\mathrm{n}}\right)$ obtendremos el intervalo de nucleación y la temperatura de máxima velocidad de nucleación (Figura 6) del vidrio procedente del lodo de El-Sadat.

Mediante DRX del vidrio original tratado térmicamente y utilizando la ecuación de Scherrer [2] podremos establecer el tamaño real de estas fases minerales desarrolladas.

En la figura 7 se observan los espectros de DRX de vidrio original tratado a $600^{\circ} \mathrm{C}$ durante 11 horas, $700,750 \mathrm{y}$ $800^{\circ} \mathrm{C}$ durante 8 horas y a $1.000^{\circ} \mathrm{C}$ durante 2 horas. En los espectros entre 600 y $800^{\circ} \mathrm{C}$ sólo aparece algunas reflexiones muy pequeñas correspondientes al inició de nucleación de la magnetita $\left(\mathrm{Fe}_{0.65} \mathrm{Fe}_{1.81} \mathrm{Mg}_{0.42} \mathrm{Al}_{0.1} \mathrm{Ti}_{0.03} \mathrm{O}_{4}\right)$. En cambio, a la temperatura de $1.000^{\circ} \mathrm{C}$ la cristalización del vidrio es total, el vitrocerámico esta formado por dos fases minerales bien cristalizadas: la magnetita (30\%) y las plagioclasas: labradoritabitownite $(70 \%)$.

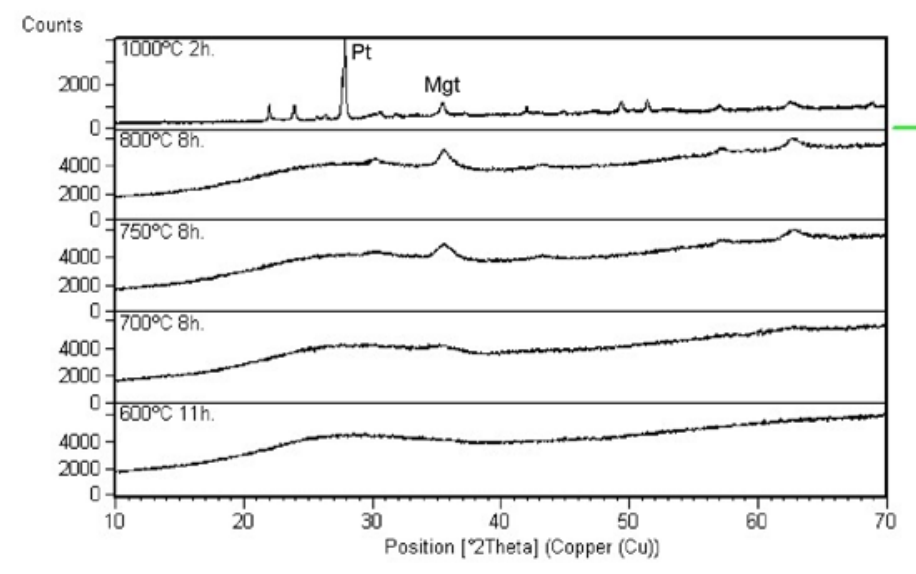

Figura 7. Espectros de difracción del vidrio tratado térmicamente a distintas temperaturas y tiempos. En el espectro correspondiente a la mayor temperatura se ha indicado el pico de maxima intensidad de las fases minerales formadas utilizando las siglas Pl para plagioclasa y Mgt para la magnetita.
El intervalo de temperatura de nucleación de la magnetita (Mgt) es de 650 a $812^{\circ} \mathrm{C}$ (Figura 6), siendo la máxima a $775^{\circ} \mathrm{C}$. Este resultado se correlaciona con las observaciones realizadas en el SEM en donde se observa que a $700^{\circ} \mathrm{C}$ solo se aprecia pequeños núcleos dispersos en la matriz vítrea, mientras que a $800^{\circ} \mathrm{C}$ se trata ya de un conjunto de cuerpos globulares y no se ve la matriz vítrea, la nucleación ha sido total (Figura 6 A y B).

Utilizando la ecuación de Scherrer:

$$
\tau=\frac{K \lambda}{\beta \cos \theta}
$$

en los difractogramas de DRX del vidrio tratado térmicamente, se ha determinado el tamaño coherente de los cristales nucleados en el vitrocerámico. En el caso de los núcleos cristalinos generados a 750 y $800{ }^{\circ} \mathrm{C}$ estos tiene un diámetro del orden de $11 \mathrm{~nm}$ y $13 \mathrm{~nm}$ respectivamente. Si se comparan estos valores con las observaciones realizadas en el SEM, se puede deducir que las esferas denominadas grandes $(80 \mathrm{~nm})$ y pequeñas $(40 \mathrm{~nm})$ en las figuras 6 A y B no corresponden a núcleos esféricos individuales pertenecientes a un solo cristal, sino que son agregados cristalinos.

La obtención del vidrio y/o del vitrocerámico a partir del lodo de la depuradora de El-Sadat es una medida medioambientalmente positiva y sostenible. La cual va en la línea de cumplir las directrices marcadas por la Unión Europea en el 6EAP. Si repasamos los puntos a los que se refiere vemos que se cumple el primero, se reducen los residuos puesto que se utilizan para fabricar un nuevo material, desarrollándose un proceso de reutilización. Paralelamente, al no tener que extraer materias primas se evita la generación de nuevos residuos. La sustitución de materias primas, y de los correspondientes productos manufacturados, por otros producidos mediante residuos eliminan la necesidad de la extracción de materias primas en una proporción equivalente a la cantidad de materia inorgánica que contienen los lodos. Considerando que añadimos un $20 \%$ de aditivos, resulta que ahorramos un $80 \%$ de materias primas y los correspondientes residuos que esta extracción generaría.

El segundo ítem habla de la reutilización de productos y componentes, en este proceso de vitrificación no tiene cabida este punto.

El tercer punto se refiere al reciclado de materiales, este se cumple totalmente en la propuesta presentada en este estudio, ya que se reciclan los lodos y a la vez se recupera el agua de humedad de los mismos (80-90\% en los lodos deshidratados). En el proceso de secado de los lodos utilizado en la actualidad el agua pasa a la atmósfera. Se recicla el material recogido en los filtros y en el tratamiento de gases durante el proceso. De manera que podemos considerar que el $95 \%$ de la composición del vidrio obtenido en el proceso de vitrificación es material reciclado.

El cuarto punto trata de la recuperación energética. En el proceso se recupera y reutiliza la energía obtenida por la combustión de la materia orgánica contenida en los lodos (un $75 \%$ ) que tienen un PCI de 13,01 MJ/Kg.

Finalmente, el quinto punto habla de fomentar un vertido ambientalmente aceptable. En el proceso de la vitrificación no se generan prácticamente residuos, de este modo se puede afirmar que en este proceso el residuo es cero. 
Si tenemos en cuenta el balance de emisión de gases, el $\mathrm{CO}_{2}$ generado en la combustión de la materia orgánica contenida en el lodo no contabiliza como $\mathrm{CO}_{2}$ de efecto invernadero ya que es una materia orgánica actual, no un combustible fósil. A la vez, la generación en el proceso de vitrificación de otros gases como el $\mathrm{NO}_{x}$ es tratado con técnicas de reducción primaria con la utilización de quemadores bajos en $\mathrm{NO}_{x^{\prime}}$ y sistemas de reacción con $\mathrm{NH}_{4}{ }^{+}$no catalizada (reducción no catalítica selectiva) de acuerdo con las Mejores Técnicas Disponibles (MTD). El $\mathrm{SO}_{3}$ es neutralizado con $\mathrm{Ca}$ $(\mathrm{OH})_{2}$ formándose $\mathrm{Ca}\left(\mathrm{SO}_{4}\right)$. Los nuevos compuestos formados en el proceso son redireccionados a la balsa de fusión con la finalidad de incorporarse de nuevo al proceso.

\section{CONCLUSIONES}

En base a la composición química de los lodos de la depuradora de El-Sadat podemos afirmar que se trata de un material que: a) es factible a ser vitrificado, solo hay que añadir una pequeña cantidad de elementos fluidificantes, para facilitar el colado del vidrio; y b) no puede utilizarse directamente en agricultura ya que contiene una proporción de metales pesados más alta que la permitida para el uso.

En el proceso de vitrificación se convierte el residuo en un sólido amorfo, se recupera calor tal y como se ha calculado con un al PCI de 13,01 MJ/Kg, se recupera agua (hasta un $80 \%$ de la inicial), a la vez que los elementos pesados se remedian, el $\mathrm{Pb}$ a las temperaturas a las que se llega en el proceso se volatiliza, y los demás pasan a formar parte de la matriz del vidrio, quedando incluidos en ella, a la vez que los posibles compuestos orgánicos se descomponen.

A $1000^{\circ} \mathrm{C}$ la cristalización del vidrio es total, se ha formado ya el vitrocerámico, el cual tendrá unas propiedades físicas de mayor resistencia que el vidrio denominado original, siendo mayor su aplicabilidad industrial, por ejemplo pudiéndose ser utilizado como pavimento.

La obtención del vitrocerámico es una alternativa tecnológica de gestión de residuos para reducir su volumen, minimizar los vertederos y valorizar el residuo, transformándolo en un producto con aplicación industrial.

\section{AGRADECIMIENTOS}

Los autores agradecen en primer lugar a los responsables de la depuradora de El-Sadat City por permitir y facilitar la recogida del lodo objeto de este estudio. Al soporte técnico de los miembros del los Servicios Científico Técnicos de la Universidad de Barcelona. La financiación del trabajo a través de los proyectos AECI-A/023061/09 y A/030032/10 que ha permitido desarrollar la recogida de las muestras, y el del Grupo Consolidado de Investigación de Recursos Minerales, 2009SGR-00444, que ha costeado los análisis.

\section{BIBLIOGRAFÍA}

(1) M.Ghazy, T. Dockhorn, N. Dichtl. Sewage Sludge Management in Egypt: Current Status and Perspectives towards a Sustainable Agricultural Use. World Academy of Science, Engineering and Technology 57, 492-500 (2009)

(2) J.A. Pérez, R. Terradas, Seijas, M.M., R. Manent, S. Martínez. Inertization of industrial wastes in ceramics materials. Ind. Ceram. 16, 7-11 (1996)

(3) A.R. Boccaccini, M. Beucker, P.A. Trusty, M. Romero, J.Ma. Rincón. Sintering behaviour of compacts made from television tube glasses. Glass Technol. 38, 128-133 (1997)

(4) J.A. Pérez, R. Terradas, M.R. Manent, S. Martinez. Sinterizzazione di scarti industriali in materiali ceramici. Ceramurgia 28, 11-14 (1998)

(5) L. Barbieri, A.C. Bonamartini, I. Lancellotti. Alkaline and alkaline-earth silicate glasses and glass-ceramics from municipal and industrial wastes. J. Eur. Ceram. Soc. 20, 2477-2483 (2000)

(6) D. Oziel Méndez Guerrero, B. A. Vázquez Méndez, A. Álvarez Méndez. Obtención de un material vitrocerámico a partir de una escoria de acería mezclada con vidrio de desecho. Bol. Soc. Esp. Ceram. Vidr., 50, 143-150 (2011)

(7) P. Colombo, G. Brusatin, E. Bernardo, G. Scarinci. Inertization and reuse of waste materials by vitrification and fabrication of glass based products. Curr. Opin. Solid St. M 7, 225-239 (2003)

(8) F.A. Corpas Iglesias, L. Pérez Villarejo, M. Benítez Guerrero, R. Artiaga Díaz, J. Pascual Cosp. Use of mud from Metallica surface treatment industrias as additive to ceramic matrices. Bol. Soc. Esp. Ceram. Vidr., 50, 117-124 (2011)

(9) M. Garcia-Valles, G. Avila, S. Martínez, R. Terradas, J.M. Nogués. Heavy metal-rich wastes sequester in mineral phases through a glass-ceramic process. Chemosphere 68, 1946-1953, (2007)

(10) S. Mestre, M.D. Palacios, M. Soriano, E. Sánchez, L. Rodríguez, J.F. Núñez, P. Rivera. Utilización de residuos industriales como opacificantes en pastas de gres porcelánico. Bol. Soc. Esp. Ceram. Vidr., 49, 297-302 (2010)

(11) M. Romero, R.D. Rawlings, J.Ma. Rincón. Crystal nucleation and growth in glasses from inorganic wastes from urban incineration. J. Non-Cryst. Solids $271,106-118(2000)$

(12) M. Romero, J.Ma. Rincón, R.D. Rawlings, A.R. Boccaccini. Use of vitrified urban incinerator waste as raw material for production of sintered glassceramics. Mater. Res. Bull. 36, 383-395 (2001)

(13) K.E.Haugsten, B. Gustavson. Environmental properties of vitrified fly ash from hazardous and municipal waste incineration. Waste Manage. 20, 167-176 (2000)

(14) T.W. Cheng, Y.S. Chen. On formation of $\mathrm{CaO}-\mathrm{Al}_{2} \mathrm{O}_{3}-\mathrm{SiO}_{2}$ glass-ceramics by vitrification of incinerator by ash. Chemosphere 51, 817-824 (2003)

(15) P. Kavouras, G. Kaimakamis, T.A. Ioannidis, T.Kehagias, P. Komninou, S. Kokkou, E. Pavlidou, I. Antonopoulos, M. Sofoniou, A. Zouboulis, C.P. Hadjiantoniou, G. Nouet, A. Prakouras, T. Karakostas. Vitrification of leadrich solid ashes from incineration of hazardous industrial wastes. Waste Manage. 23, 361-371 (2003)

(16) T.W. Cheng, M.Z. Huang, C.C. Tzeng, K.B. Cheng, T.H. Ueng. Production of coloured glass-ceramics from incinerator ash using thermal plasma technology. Chemosphere 68, 1937-1945 (2007)

(17) C.H. Jung, T. Matsuto, N. Tanaka. Behavior or metals in ash melting and gasification-melting of municipal solid waste (MSW). Waste Manage. 25, 301-310 (2005).

(18) V.I. Davydov, V.P. Burdinskii, P.G. Dobrygin. Equipment for vitrification of nuclear power plant wastes in a direct-heating ceramic furnace. Atom. Energy 80, 219-221 (1996)

(19) L.S. Pioro, B.F. Sadovskiy, I.L. Pioro. Research and development of a high-efficiency one-stage melting converter-burial-bunker method for vitrification of high-level radioactive wastes. Nucl. Eng. Des. 205, 133-144 (2001)

(20) R.W. Le Maitre, A. Streckeisen, B. Zanettin, M. J. Le Bas, B. Bonin, P. Bateman, G. Bellieni, A. Dudek, S. Efremova, J. Keller, J. Lamere, P.A. Sabine, R. Schmid, H. Sorensen, A.R. Woolley. Igneous Rocks: A Classification and Glossary of Terms: Recommendations of the International Union of Geological Sciences, Subcommission on the Systematics of Igneous Rocks, 2nd edition, ed. by R.W. Le Maitre,. Cambridge University Press, Cambridge, U.K., New York, 236 p. (2002)

(21) BOE (Boletín Oficial del Estado). Núm. 262, Real Decreto 1310/1990, España (1990)

(22) CEE. Diario Oficial de las Comunidades Europeas. N ${ }^{\circ}$ L 181/10 (1986)

(23) J.Xu Xiaojie, S.Ray Chandra, E.Day Delbert. Nucleation and Cystallization of $\mathrm{Na}_{2} \mathrm{O} .2 \mathrm{CaO} .3 \mathrm{SiO}_{2}$ Glass by Differential Termal. Analysis. J.Am.Cer.Soc. 74, 909-914 (1990)

(24) P. Scherrer. Göttinger Nachrichten Gesell., Vol. 2, p 98 (1918) 